\title{
AZACITIDINE TREATMENT IN PATIENTS WITH MYELODYSPLASTIC SYNDROME, CHRONIC MYELOMONOCYTIC LEUKEMIA TYPE 2 AND ACUTE MYELOID LEUKEMIA ACCORDING TO THEIR CYTOGENETIC FINDINGS
}

\author{
D. Nikolova ${ }^{*}$, A. Yordanov ${ }^{2 *}$, A. Radinov ${ }^{2}$ \\ ${ }^{1}$ Department of Medical Genetics, Medical University - Sofia, Bulgaria \\ 2University Hospital for Active Treatment "Sv. Ivan Rilski" - Sofia, Bulgaria
}

\begin{abstract}
Introduction: Azacitidine is one of the hypomethylating agents available for the treatment of elderly patients with myelodysplastic syndrome (MDS) or acute myeloid leukemia (AML). It is also used as an appropriate treatment of chronic myelomonocytic leukemia (CMML) in the real life setting. As treatment of $A M L$ and $C M M L$ is not curative, and allogeneic stem cell transplantation (allo-SCT) remains traditionally the only option, significant clinical benefits by hypomethylating agents have been reported. According to the available data, $16 \%$ of subjects with MDS who received azacitidine had a complete or partial normalization of blood cell counts and bone marrow morphology, while two-thirds of patients who required blood transfusions no longer needed them. Nevertheless, it can also be hepatotoxic in patients with severe liver impairment and extensive liver tumors. Aim: to summarize the effect of azacitidine treatment in patients in the light of their general condition, blood count parameters, toxicity (general and hematologic), as well as the presence of cytogenetic aberrations. Materials and Methods: Twenty-seven patients of which 15 patients with MDS, 9 patients with CMML and 3 patients with AML received azacitidine treatment. The blood count levels and toxicity were followed for a period of twelve months. Results: $22.2 \%$ of our patients ( 6 of 27) of different hematologic diagnoses showed genetic aberrations in their DNA. All they showed quick disease progression and fatal outcome, four of them also developed hematologic toxicity. The remaining $77.8 \%$ had no cytogenetic findings. Of all the cohort, $19.05 \%$ developed toxicity during the course of the treatment, $38 \%$ - decreased leucocyte levels, $14.3 \%$ - decreased thrombocyte levels and $18.2 \%$ decreased hemoglobin level. The erythrocyte levels were not substantially influenced by the treatment. The majority of the patients sustained stable levels of red blood cells, as well as of platelets and hemoglobin without remarkable changes between month 0 and month 6 of the treatment. Conclusion: Our results showed, that the main disadvantage of azacitidine treatment in our patients were progressive leucopenia (in 10/27 patients or $37 \%$ of cases) and toxicity ( $8 / 27$ or $29.6 \%$ of cases).
\end{abstract}

Key words: Azacitidine (AZA, Vidaza $\left.{ }^{\circledR}\right)$, monitoring, blood count parameters, toxicity, cytogenetic findings

Corresponding author: Assist. Prof. Dragomira Nikolova, PhD, Department of Medical Genetics, Medical University - Sofia, Bulgaria, e-mail: dmb@abv.bg

RECEIVED: 29 January 2021; ACCEPTED: 6 February 2021 


\section{INTRODUCTION}

Myelodysplastic syndromes (MDS) are a heterogeneous group of clonal stem cell disorders with a tendency for leukemic transformation. Diagnosis is currently based on the presence of peripheral blood cytopenias, peripheral blood and bone marrow dysplasia/blasts, and clonal cytogenetic abnormalities [1]. The International Prognostic Scoring System (IPSS-R) has been generally accepted for risk stratification and prognosis of the disease. Depending on the IPSS-R risk group, the median survival time of the patients is quite short - from 8 months in the very high risk group to a little longer than eight years in the very low risk group [2]. Hypomethylating agents (HMA), such as azacitidine, decitabine and lenalidomide for patients with isolated $\operatorname{del}(5 q)$ aberration, have been approved and used as treatment options for MDS, however with limited effect on recovery and survival [1]. Azacitidine (Vidaza) was synthesized as a potential chemotherapeutic agent for cancer and may lead to a complete or partial normalization of blood cell counts and bone marrow morphology in $16 \%$ of subjects with MDS who were randomized to receive it [2]. In addition, about two-thirds of patients who required blood transfusions no longer needed them after receiving azacitidine [3]. It has become a standard therapy for high-risk myelodysplastic syndromes (MDS) and elderly patients with acute myeloid leukemia (AML) $[4,5]$. It is also an option for patients with CMML 2 that can provide patient free survival and overall survival for approximately one year irrespective of age or disease subgroup [6, 7]. Azacitidine exhibits its antineoplastic activity via two mechanisms - by hypomethylation of DNA (inhibiting of DNA methyltransferase), and by direct cytotoxicity on abnormal hematopoietic cells in the bone marrow through its incorporation into DNA and RNA. Chemically, AZA is a ribonucleoside, so it is incorporated into RNA to a larger extent than into DNA.

Except its demonstrated favorable effects, Vidaza $₫$ exhibits negative side effects, causing mostly anemia, neutropenia, and thrombocytopenia. So the patients should be frequently monitored, more specifically prior to each dosing cycle. The dose may have to be adjusted based on nadir counts and hematologic response. In addition, the drug can also be hepatotoxic in patients with severe liver impairment, and patients with extensive liver tumors who could develop progressive hepatic coma and death during azacitidine treatment, especially if albumin levels less than $30 \mathrm{~g} / \mathrm{L}$.

\section{MATERIALS AND METHODS}

\section{Participants in the study}

Twenty-seven patients included in this study were admitted to the Unit of Clinical Hematology, General Hospital for Active Treatment "St. Ivan Riski", Sofia, Bulgaria. All patients signed a written informed consent for treatment with Vidaza ${ }^{\circledR}$ at a dose specified by their medical doctor. Decisions about the duration of the treatment period were also taken by their physician. All patients gave consent for their results to be reported, discussed and shared keeping their personal data confidential. Information regarding previous treatment decision of each patient, and values like age, sex and clinical diagnosis, was also available.

\section{Clinical characteristics of the patients}

A total of twenty-seven patients were included and their results - retrospectively reviewed. The mean age of the patients was $68(55-82)$ years. The distribution by gender was as follows: $18(67 \%)$ males and $9(33 \%)$ females. Patients diagnosed with MDS (15) were risk stratified according to the Revised International Prognostic Scoring System (IPSS-R) [8]. Five of them were classified as MDS-RAEB 1 (Refractory Anaemia with Excess Blasts, type 1), ten - RAEB 2 (Refractory Anaemia with Excess Blasts, type 2). Nine patients were diagnosed with CMML 2, other three - with AML. Twenty-two patients received azacitidine as a first-line treatment, while the remaining five - as a second-line therapy. The initial dose for all the patients started between 100-150 mg/D1D7 upon their physician's decision (Table 1).

\section{Laboratory tests}

Whole blood count (WBC) tests were performed for all patients before treatment, as well as during the treatment at three month' periods (first, third, sixth, ninth and twelfth month). Some data is missing at the end of the monitored period because of inevitable circumstances (either the patient had passed away or had decided to stop the treatment). The main parameters analyzed in this study were: WBC count (white blood cell), RBC (red blood cell) and PLT (platelets), as well as the hemoglobin level $(\mathrm{Hb})$. Toxicity (hematologic and other) was also assessed and taken in consideration affecting the treatment dose, its reduction, or the eventual treatment discontinuation.

\section{Cytogenetic analysis}

Cytogenetic analysis of each patient was performed by standard karyotyping. Karyotypes were analyzed and they presented in Table 2. 
Table 1. Clinical characteristics of the monitored patients - age, sex, diagnosis (according to IPSS-R system), risk stratification, previous treatment and initial dose of azacitidine

\begin{tabular}{|c|c|c|c|c|c|c|}
\hline & Age & Sex & Diagnosis & Risk stratification & Previous treatment & Vidaza dose mg/D1-D7 \\
\hline 1 & 82 & $\mathrm{~F}$ & MDS-RAEB 1 & 2 & no & 100 \\
\hline 2 & 55 & $\mathrm{~F}$ & MDS-RAEB 1 & 3 & no & 120 \\
\hline 3 & 67 & $\mathrm{M}$ & MDS-RAEB 2 & 4 & no & 150 \\
\hline 4 & 72 & $\mathrm{M}$ & MDS-RAEB 2 & 4,5 & no & 140 \\
\hline 5 & 58 & M & MDS-RAEB 1 & 5 & no & 150 \\
\hline 6 & 60 & $M$ & MDS-RAEB 2 & 5 & no & 150 \\
\hline 7 & 76 & $\mathrm{~F}$ & MDS-RAEB 2 & 5 & no & 120 \\
\hline 8 & 81 & M & MDS-RAEB 1 & 5 & no & 100 \\
\hline 9 & 58 & $M$ & MDS-RAEB 2 & 5,5 & no & 145 \\
\hline 10 & 74 & $\mathrm{~F}$ & MDS-RAEB 2 & 6 & no & 129 \\
\hline 11 & 58 & M & MDS-RAEB 1 & 6 & no & 150 \\
\hline 12 & 72 & M & MDS-RAEB 2 & 6,5 & no & 100 \\
\hline 13 & 69 & $F$ & MDS-RAEB 2 & 6,5 & yes & 100 \\
\hline 14 & 73 & $\mathrm{~F}$ & MDS-RAEB 2 & 7 & no & 100 \\
\hline 15 & 58 & $\mathrm{~F}$ & MDS-RAEB 2 & 8 & no & 100 \\
\hline 16 & 56 & $M$ & CMML 2 & $\mathrm{NA}$ & no & 150 \\
\hline 17 & 78 & $M$ & CMML 2 & NA & no & 100 \\
\hline 18 & 57 & M & CMML 2 & NA & no & 150 \\
\hline 19 & 61 & M & CMML 2 & $\mathrm{NA}$ & yes & 150 \\
\hline 20 & 64 & M & CMML 2 & NA & no & 140 \\
\hline 21 & 69 & $\mathrm{~F}$ & AML M5A & NA & no & 133 \\
\hline 22 & 72 & $\mathrm{~F}$ & CMML 2 & NA & yes & 100 \\
\hline 23 & 75 & $M$ & CMML 2 & NA & yes & 140 \\
\hline 24 & 75 & M & AML M5A & NA & no & 145 \\
\hline 25 & 78 & M & CMML 2 & NA & no & 150 \\
\hline 26 & 79 & $M$ & CMML 2 & NA & no & 100 \\
\hline 27 & 59 & $M$ & AML M2 & NA & yes & 100 \\
\hline
\end{tabular}

${ }^{*}$ MDS-RAEB 1(Refractory Anaemia with Excess Blasts, type 1), MDS-RAEB 2 (Refractory Anaemia with Excess Blasts, type 2), CMML 2 (Chronic myelomonocytic leukemia), AML M5A (Acute monoblastic leukemia (M5a)), AML M2 (Acute myeloblastic leukemia with maturation (M2)); NA - data not available

Table 2. Toxicity and total number of white blood cells (WBC), PLT (platelets), Hb (hemoglobin) and erythrocytes (RBC) in patients with cytogenetic aberrations

\begin{tabular}{|c|c|c|c|c|c|c|c|c|c|c|c|c|c|c|c|}
\hline 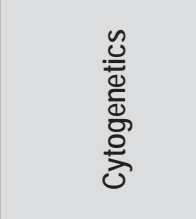 & $\begin{array}{l}\frac{n}{n} \\
\frac{0}{0} \\
\frac{\pi}{0} \\
\frac{\pi}{0}\end{array}$ & 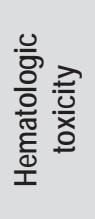 & $\begin{array}{l}\frac{7}{0} \\
\frac{0}{x} \\
\frac{0}{2} \\
\frac{0}{0} \\
\frac{0}{0}\end{array}$ & 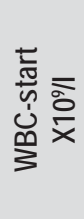 & 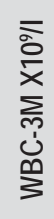 & 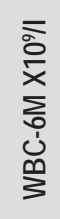 & 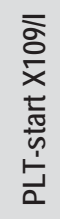 & 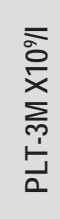 & 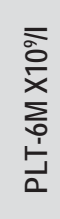 & 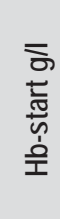 & 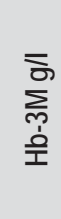 & $\begin{array}{l}\overline{0} \\
\sum_{0} \\
\text { 이 }\end{array}$ & 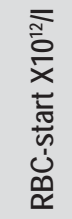 & 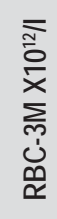 & 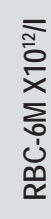 \\
\hline $\begin{array}{l}\text { 45,X,-Y; del } \\
(1,15)(p 10, q 10)\end{array}$ & CMML 2 & yes & no & 34,4 & 5,2 & NA & 338 & 38 & NA & 105 & 113 & NA & 3,02 & 3,9 & NA \\
\hline $46, X X, 1 q h+$ & MDS-RAEB 2 & yes & yes & 2,3 & 8,2 & NA & 30 & 85 & NA & 70 & 62 & NA & 3,11 & 2,2 & NA \\
\hline $45, X$ & MDS-RAEB 2 & yes & no & 6,7 & 3,7 & 4,8 & 74 & 93 & 79 & 96 & 76 & 98 & 2,8 & 2,5 & 3,2 \\
\hline $47,+8$ & AML M5A & no & no & 11,9 & NA & NA & 22 & NA & NA & 158 & NA & NA & 6,0 & NA & NA \\
\hline complex & AML M2 & yes & yes & 1,9 & NA & NA & 77 & NA & NA & 70 & NA & NA & 2,5 & NA & NA \\
\hline $\operatorname{del}(7 q)$ & MDS-RAEB 1 & no & no & 4,6 & 5,8 & 8,7 & 127 & 150 & 102 & 69 & 71 & 69 & 2,2 & 2,6 & 2,5 \\
\hline
\end{tabular}

* WBC-baseline (white blood cells' count at basline); WBC-3M (white blood cells' count at the end of the third month of treatment); WBC-6M (white blood cells' count at the end of the sixth month of treatment); PLT - baseline (platelets' count measured at the beginning of the treatment); PLT-3M (platelets count at the end of the third month of the treatment); PLT$6 \mathrm{M}$ (platelets count at the end of the sixth month of the treatment); $\mathrm{Hb}$ - baseline (level of hemoglobin at the beginning of the treatment); Hb-3M (level of hemoglobin at the end of the third month of the treatment); Hb-6M (level of hemoglobin at the end of the sixth month of the treatment); RBC-baseline (erythrocytes' number at the beginning of the treatment); RBC$3 \mathrm{M}$ (erythrocytes' number at the end of the third month of the treatment); RBC-6M (erythrocytes' number at the end of the sixth month of the treatment); NA - data not available 


\section{RESULTS}

\section{Patients with cytogenetic aberrations}

Cytogenetic aberrations were detected by standard karyotyping in 6 patients (6/27 or $22.2 \%)$. The aberrations were quite heterogeneous: one patient displayed a complex karyotype ( $\geq 3$ cytogenetic abnormalities); one - an additional chromosome 8; one was with partial deletion of chromosome 7 (deletion $7 q)$, one - with a polymorphic region in chromosome 1q (1qh+). Two subjects displayed a loss of a sex chromosome ( $\mathrm{Y}$ in a male patient and an $\mathrm{X}$ chromosome in a female one) (Fig. 1). Three patients were with MDS (stratified as high risk group, scoring 6, 6.5 and 8 respectively), two were with AML, and one with CMML 2. The mean age of patients with cytogenetic aberrations in the karyotype was 67.5 years (58-78 years), distribution by sex was four males vs. two females, and one of them had changed to AML after the initiation of the treatment with Vidaza $®$. Four of the six patients developed hematologic toxicity $(67 \%)$, in two of them it was accompanied by other type of toxicity. During the course of the therapy, two patients experienced decreased mean leucocytes and platelets. All of them maintained stable levels of hemoglobin and erythrocytes (Table 2).

\section{Patients without cytogenetic aberrations}

No chromosomal aberrations were detected in the majority of patients (21 out of 27 patients, Figure 1), mean age 68 years (55-82 years), 14 males, 7 females, neither has progressed to AML. Nine patients had CMML 2, one - AML, other twelve were MDS positive, stratified according to IPSS-R variably, from a low to a high risk group.

Upon treatment with AZA only four participants had developed toxicities $(4 / 21,19 \%)$ (Table 3$)$. During the six month monitoring eight patients $(38 \%)$ developed leucopenia with their average WBC count decreasing by at least $30 \%$. Other patients had stabilized levels of WBC and even slightly increased ones. Thrombocytopenia was rarely displayed in this group (three patients had decreased PLT counts; 14.3\%), the number of RBC was generally stable yet two patients developed anemia (hemoglobin levels decreased).

\section{Whole study group}

Eight of our 27 patients (8/27 or $29.6 \%$ ) experienced a toxic effect during the treatment, in two of them the effect was additive as hematologic toxicity was accompanied by another type also. Leucopenia was the most common side effect ocurring in 10 patients $(37 \%)$, while thrombocytopenia and anemia were rarer events (in $5 / 27,18.5 \%$ and $2 / 37,7.4 \%$ respectively). At treatment end, the outcome was fatal for six patients, while the remaining 21 continued their therapeutic regimen. Generally, the six-month survival rate was around $78 \%$. On overall, five patients discontinued the treatment and two of them - because of the occurrence of hematologic toxicity.

\section{DISCUSSION}

Azacitidine, the first drug approved by FDA for the treatment of MDS, has a favorable safety profile and provides a clinical benefit of eliminating transfusion dependence and complete or partial normalization of blood counts and bone marrow blast percentages in responding patients [3]. Its mechanism of action in myelodysplastic syndromes is still not fully understood. Probably, as a DNA-methyltransferase inhibitor, azacitidine restores normal transcription of tumor suppressor genes and counteracts DNA hypermeth-

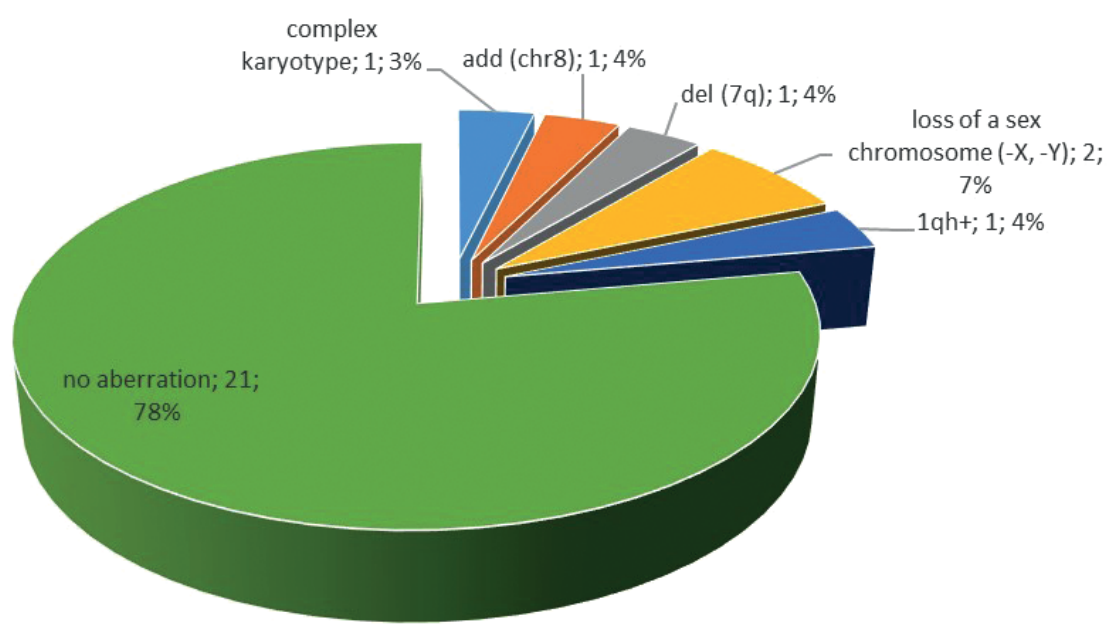

Fig. 1. Distribution of patients according to the identified mutations in their karyotype 
Table 3. Toxicity and total number of white blood cells (WBC), PLT (platelets), Hb (hemoglobin) and erythrocytes (RBC) in patients without cytogenetic aberrations

\begin{tabular}{|c|c|c|c|c|c|c|c|c|c|c|c|c|c|c|}
\hline $\begin{array}{l}\text { 을 } \\
\frac{0}{0} \\
\frac{0}{0} \\
\frac{0}{5}\end{array}$ & 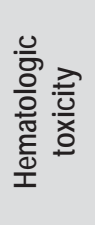 & 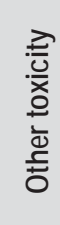 & 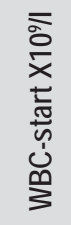 & 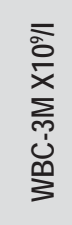 & 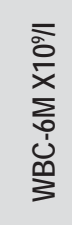 & 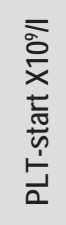 & 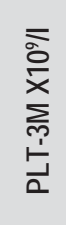 & 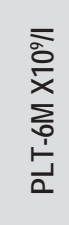 & 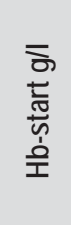 & $\begin{array}{l}\bar{\delta} \\
\sum_{\text {mo }} \\
\text { 全 }\end{array}$ & $\begin{array}{l}\bar{\sigma} \\
\sum_{0}^{0} \\
\text { 京 }\end{array}$ & 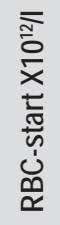 & 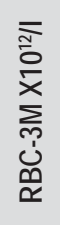 & 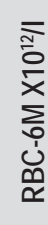 \\
\hline normal & no & no & 8,6 & 3,7 & 5,2 & 180 & 220 & 213 & 134 & 135 & 141 & 4,5 & 4,3 & 4,9 \\
\hline normal & no & no & 13,9 & 10,3 & 5,5 & 177 & 267 & 273 & 162 & 165 & 160 & 6,4 & 6,5 & 6,0 \\
\hline normal & no & yes & 17,2 & 21,2 & 13,3 & 99 & 155 & 163 & 105 & 109 & 104 & 3,6 & 3,5 & 3,1 \\
\hline normal & no & no & 17,3 & 5,6 & 4,9 & 535 & 735 & 410 & 79 & 77 & 77 & 3,0 & 2,8 & 2,8 \\
\hline normal & no & no & 4,6 & 5,8 & 9,7 & 127 & 150 & 162 & 69 & 71 & 80 & 2,2 & 2,6 & 2,9 \\
\hline normal & no & no & 2,7 & 4,8 & 7,4 & 57 & 239 & 247 & 102 & 124 & 136 & 2,9 & 4,0 & 5,0 \\
\hline normal & no & no & 7,0 & NA & NA & 63 & NA & NA & 140 & NA & NA & 5,5 & NA & NA \\
\hline normal & no & no & 17,3 & 6,1 & 5,2 & 201 & 337 & 358 & 123 & 123 & 125 & 3,2 & 3,5 & 3,6 \\
\hline normal & no & no & 2,9 & 4,3 & 4,0 & 127 & 119 & 167 & 89 & 121 & 116 & 2,4 & 3,8 & 3,8 \\
\hline normal & no & no & 2,2 & 3,6 & 44,4 & 121 & 98 & 46 & 101 & 88 & 82 & 3,5 & 3,3 & 3,0 \\
\hline normal & no & no & 5,2 & 5,8 & 4,8 & 75 & 92 & 135 & 124 & 134 & 145 & 4,1 & 5,4 & 5,7 \\
\hline normal & yes & no & 2,2 & 5,4 & NA & 76 & 358 & NA & 60 & 68 & NA & 1,6 & 2,2 & NA \\
\hline normal & no & no & 20,4 & 4,3 & 5,5 & 44 & 106 & 144 & 79 & 105 & 108 & 2,8 & 3,9 & 4,0 \\
\hline normal & yes & no & 2,6 & NA & NA & 13 & NA & NA & 68 & NA & NA & 2,5 & NA & NA \\
\hline normal & no & no & 5,1 & 3,6 & NA & 292 & 346 & NA & 85 & 69 & NA & 3,2 & 2,3 & NA \\
\hline normal & no & no & 40,5 & 16,0 & 4,5 & 216 & 286 & 263 & 101 & 97 & 106 & 3,8 & 4,2 & 4,4 \\
\hline normal & no & no & 2,0 & 3,1 & NA & 146 & 332 & NA & 82 & 82 & NA & 2,4 & 2,5 & NA \\
\hline normal & no & no & 6,7 & 7,8 & 10,0 & 66 & 3 & 179 & 143 & 129 & 136 & 3,9 & 3,3 & 3,8 \\
\hline normal & no & no & 36,9 & 8,9 & 6,1 & 71 & 117 & 237 & 91 & 112 & 83 & 3,0 & 3,7 & 3,0 \\
\hline normal & yes & no & 5,0 & 3,9 & 5,0 & 159 & 191 & 123 & 94 & 120 & 80 & 2,6 & 3,2 & 2,4 \\
\hline normal & no & no & 9,4 & 32,2 & 10,4 & 11 & 35 & 80 & 95 & 89 & 94 & 3,1 & 2,9 & 2,8 \\
\hline
\end{tabular}

* WBC-baseline (white blood cells' count at the beginning of the treatment); WBC-3M (white blood cells' count at the end of the third month of the treatment); WBC-6M (white blood cells' count at the end of the sixth month of the treatment); PLT baseline (platelets' count measured at the beginning of the treatment); PLT-3M (platelets'count at the end of the third month of the treatment); PLT-6M (platelets'count at the end of the sixth month of the treatment); Hb - baseline (level of hemoglobin at the beginning of the treatment); $\mathrm{Hb}-3 \mathrm{M}$ (level of hemoglobin at the end of the third month of the treatment); Hb-6M (level of hemoglobin at the end of the sixth month of the treatment); RBC - baseline (erythrocytes' number at the beginning of the treatment); RBC-3M (erythrocytes' number at the end of the third month of the treatment); RBC-6M (erythrocytes' number at the end of the sixth month of the treatment); NA - data not available

ylation, which has been implicated in the progression of myelodysplastic syndromes [9, 10].

During randomized clinical trials of azacitidine, abnormal karyotypes had been detected in 45 to $51 \%$ of patients with MDS, while complex karyotypes - in 9 to $11 \%$ [1, 11]. In an earlier study of a Bulgarian group including a similar number of patients (27), cytogenetic abnormalities were detected in eleven of them or $40.7 \%$ (6 with complex karyotype, 5 with other chromosomal mutations), while 16 presented a normal karyotype [5]. In our study, $22.2 \%$ of patients had cytogenetic aberrations, and one had a complex karyotype (3.7\%). This can be due either to the low frequency of cytogenetic mutations in our patients, or to the small number of patients in the cohort. Among MDS cases, the most common cytogenetic abnormalities were considered to be del (5q), followed by trisomy $8,-Y$, del (20q), and monosomy $7[11,12]$. The $5 q$ deletion invariably affects the q31 to q33 bands [1]. Yet in our patients, karyotype 46,XX,del(5q) or $46, \mathrm{XY}$, del $(5 q)$ was not detected. Nevertheless, Varbanova et al. reported del $(5 q)$ in one patient with a good prognostic risk [6]. We report additional chromosome 8 and a partial deletion of chromosome 7 (deletion 7q) in two separate cases (Table 2). The deletion of chromosome 7 was found in two patients by Mincheva et al. [5]. Yet none of them has found a loss of sex chromosomes. Loss of chromosome $Y$ 
is found in healthy elderly males and could be present as a rare finding in MDS cases [13]. In our study, deletion of $Y$ was detected in one patient; another female patient has lost one of the sex chromosomes $(45, X)$. Additionally, we detected a translocation between chromosomes 1 and 15, and a polymorphic region in chromosome 1 (Table 2).

Even if karyotypic abnormalities are expected to be common in MDS, treatment with azacitidine has demonstrated prolonged overall survival regardless of the IPSS cytogenetic risk category [14]. In addition, the drug showed effectiveness in higher-risk MDS patients with both complex or non-complex karyotypes. Fenaux $\mathrm{P}$ et al. showed that patients with unfavorable cytogenetics demonstrated poor survival when treated with low-dose cytarabine compared to azacitidine [15]. On the other hand, azacitidine is reported as a preferred treatment for older patients with AML with adverse-risk cytogenetics, particularly those with chromosome 5, 7, and/or 17 abnormalities and complex or monosomal karyotypes [16]. In our study, patients with cytogenetic aberrations developed higher toxicity $(67 \%)$ following AZA treatment compared to patients without cytogenetic abnormalities (toxicity in this group around 19\%). We cannot comment here the role of each cytogenetic aberration independently because of the low number of patients enrolled. However, according to our results, toxicity due to treatment depends on karyotype mutations which remain an unfavorable factor for the treatment response.

There are controversial opinions about the duration of azacitidine treatment needed for the improvement of the patients. A study of 38 patients on azacitidine treatment reported that long-term treatment with azacitidine was necessary for an optimal benefit [17]. According to others, patients may benefit even from a limited number of courses [18]. Out of 32 patients receiving 5-AZA, complete remissions were achieved in $15.6 \%$ and stable disease in $34.4 \%$ of patients. The most common adverse event of azacitidine treatment in a phase III clinical trial was peripheral cytopenia [15]. Peripheral blood counts normalized in $6.3 \%$ of patients while hematologic improvement was achieved in $25 \%$ [18]. In our cohort, the strongest side effects of AZA treatment remained toxicity (30\% of patients) and leucopenia (37\% of patients). The rates of thrombocytopenia $(18.5 \%)$ and anemia (only $7 \%$ ) were lower. These data are consistent with the findings of other authors (Varbanova et al.) who reported the most frequent adverse events being neutropenia $38.5 \%$, followed by anemia $15.4 \%$ and thrombocytopenia $11.5 \%$ [6].

Because of these high rates of leucopenia and drug toxicity, some authors state that intensive chemother- apy might remain the appropriate treatment in some situations in higher-risk myelodysplastic syndromes, especially in candidates with excess of marrow blasts and an unfavorable karyotype [15]. Anyway, in a small number of patients like ours it is really difficult to decide on whether the demonstrated cytopenia is due to the possible myelotoxicity of AZA, or reflects the activity of the myelodysplasia itself. The high percentage of cases with leucopenia and toxicity in our study remains to be clarified in larger studies. Generally, demethylating agents (DMA) like AZA, are still used as promising treatment options for patients with MDS [19]. In spite of the unfavourable side effects, the drug continues to be recommended as the first-line hypomethylating agent for elderly patients or those with high risk MDS, AML and CMML [20].

\section{CONCLUSION}

Our observational study showed that patients with cytogenetic aberrations may benefit less from AZA treatment, but this could be due to the quick disease progression in those patients. For the whole cohort of 27 patients, the levels of RBC remained stable, without remarkable changes between month 0 and month 6 of treatment. Thrombocytopenia and anemia were common within lower range levels $(18.5 \%$ and $7 \%$ respectively). Our main concern remains leucopenia ( $37 \%$ of patients) and toxicity ( $30 \%$ of patients). Therefore, we recommend the application of Vida$\mathrm{za} \otimes$ with caution and taking into consideration the patients' condition and specific response to therapy.

Disclosure summary: The authors have nothing to disclose.

Acknowledgements: *The authors contributed equally to this manuscript.

\section{REFERENCES}

1. Gangat N, Patnaik M, Tefferi A. Myelodysplastic syndromes: Contemporary review and how we treat. Am J Hematol. 2016; 91(1): 76-89.

2. Cihak A. Biological effects of 5 -azacytidine in eukaryotes. Oncology. 1974; 30(5): 405-22.

3. Kaminskas E, Farrell A, Wang Y, et al. FDA drug approval summary: azacitidine (5-azacytidine, Vidaza) for injectable suspension. Oncologist. 2005;10(3):176-82.

4. Estey E. Epigenetics in clinical practice: the examples of azacitidine and decitabine in myelodysplasia and acute myeloid leukemia. Leukemia. 2013; 27(9):1803-12.

5. Micheva I, Dimitrova S, Efraim M, et al. Outcome after azacitidine treatment in patients with high-risk myelodysplastic syndrome and acute myeloid leukemia in the Clinic of Hematology at St. Marina University Hospital, Varna. Scripta Scientifica Medica. 2018; 50(1):31-35

6. Varbanova V, Anastasova-Postadzhiyan A, Nedeva A, et al. Outcome After Azacitidine Treatment in Patients with High- 
Risk Myeolodysplastic Syndrome, Chronic Myelomonocytic Leukemia Type 2 and Acute Myeloid Leukemia - A Single Center Experience (Preliminary Data). Acta Medica Bulgarica. 2020;47:38-43.

7. Tendas A, Cupelli L, Siniscalchi A, et al. Azacitidine in chronic myelomonocytic leukemia: an effective and manageable approach. Mediterr J Hematol Infect Dis. 2014;6(1): e2014020.

8. Della Porta MG, Tuechler H, Malcovati L, et al. Validation of WHO classification-based Prognostic Scoring System (WPSS) for myelodysplastic syndromes and comparison with the revised International Prognostic Scoring System (IPSS-R). A study of the International Working Group for Prognosis in Myelodysplasia (IWG-PM). Leukemia. 2015;29(7):1502-13.

9. Raj K, John A, Ho A, et al. CDKN2B methylation status and isolated chromosome 7 abnormalities predict responses to treatment with 5-azacytidine. Leukemia. 2007; 21(9): 1937-44.

10. Gore S, Baylin S, Sugar E, et al. Combined DNA methyltransferase and histone deacetylase inhibition in the treatment of myeloid neoplasms. Cancer Res. 2006;66(12): 6361-9.

11. Schanz J, Tuchler H, Sole F, et al. New comprehensive cytogenetic scoring system for primary myelodysplastic syndromes (MDS) and oligoblastic acute myeloid leukemia after MDS derived from an international database merge. $\mathrm{J}$ Clin Oncol. 2012;30(8):820-9.

12. Gangat N, Patnaik M, Begna K, et al. Primary Myelodysplastic Syndromes: The Mayo Clinic Experience With $1000 \mathrm{~Pa}-$ tients. Mayo Clin Proc. 2015;90(12):1623-38.
13. Wiktor A, Rybicki B, Piao ZS, et al. Clinical significance of $Y$ chromosome loss in hematologic disease. Genes Chromosomes Cancer. 2000;27(1):11-6.

14. Mufti G, Gore S, Santini V, et al. Influence of Karyotype On Overall Survival in Patients with Higher-Risk Myelodysplastic Syndrome Treated with Azacitidine or a Conventional Care Regimen. Blood. 2009;114(22):1755.

15. Fenaux P, Mufti G. J, Hellstrom-Lindberg E, et al. Efficacy of azacitidine compared with that of conventional care regimens in the treatment of higher-risk myelodysplastic syndromes: a randomised, open-label, phase III study. Lancet Oncol. 2009;10(3):223-32.

16. Dohner H, Dolnik A, Tang L, et al. Cytogenetics and gene mutations influence survival in older patients with acute myeloid leukemia treated with azacitidine or conventional care. Leukemia. 2018;32(12):2546-2557.

17. Usami E, Kimura M, Takenaka $\mathrm{S}$ et al. Continuity and efficacy of real-world use of azacitidine. Pharmazie. 2020;75(4):154-158.

18. Muller-Thomas $C$, Schuster T, Peschel $C$, et al. A limited number of 5-azacitidine cycles can be effective treatment in MDS. Ann Hematol. 2009;88(3):213-9.

19. Cseh A.M, Niemeyer C. M, Yoshimi A et al. Therapy with low-dose azacitidine for MDS in children and young adults: a retrospective analysis of the EWOG-MDS study group. $\mathrm{Br} \mathrm{J}$ Haematol. 2016;172(6):930-6.

20. Xie M, Jiang Q, Xie $Y$ et al. Comparison between decitabine and azacitidine for the treatment of myelodysplastic syndrome: a meta-analysis with 1,392 participants. Clin Lymphoma Myeloma Leuk. 2015;15(1):22-8. 